\title{
TVT: 3 AÑOS DE EXPERIENCIA
}

\author{
J. JIMÉNEZ CALVO, A. HUALDE ALFARO, A. SANTIAGO GONZÁLEZ DE GARIBAY, \\ M. PINÓS PAUL, J. JIMÉNEZ ARISTU, M. MONTESINO SEMPER, \\ A. DE PABLO CÁRDENAS, F. LOZANO URUÑUELA, L. RIPA SALDIAS
}

Servicio de Urología. Hospital Virgen del Camino. Pamplona. Navarra.

Actas Urol Esp. 28 (1): 13-20, 2004

\section{RESUMEN}

TVT: 3 AÑOS DE EXPERIENCIA

INTRODUCCIÓN: Desde la descripción de la técnica de TVT para el tratamiento de la incontinencia urinaria de esfuerzo en 1996, se han colocado unas 150.000 unidades. En noviembre de 1998 iniciamos esta técnica en nuestro servicio, en este artículo analizamos nuestros casos en estos tres años.

MATERIAL Y MÉTODOS: Hemos intervenido a 142 pacientes, con una edad media de 59 años. En el 57\% de ellas, además de realizar TVT, asociamos reparación de defectos anatómicos pélvicos.

RESULTADOS: Con un seguimiento medio de 17 meses y mediana de 14 meses el 93\% de las pacientes están curadas. Los fracasos aparecieron de forma precoz en los 6 primeros meses de seguimiento. Como complicaciones aparecieron un 4,8\% de perforaciones vesicales, retenciones post-operatorias de menos de 30 días en el 17\%, retenciones a largo en el 2\%, hematomas post-quirúrgicos en el 2,7\% y urgencia de novo en el 9\%. La cirugía se realizó en 10 pacientes con antecedentes de cirugía pelviana anti-incontinencia con buenos resultados en todos los casos.

CONCLUSIONES: Es una técnica quirúrgica sencilla, que requiere un corto tiempo quirúrgico, y que puede ser realizada en régimen de cirugía mayor ambulatoria. Es una técnica no exenta de complicaciones aunque la proporción de ellas es baja y con unos resultados que siguen siendo alentadores (aunque debemos esperar que nuestros estudios a largo plazo coincidan con los ya publicados con tasas de éxito del 84,7\%).

PALABRAS CLAVE: TVT. Incontinencia urinaria de esfuerzo.

\section{ABSTRACT}

TVT: THREE YEARS OF EXPERIENCE

INTRODUCTION: Since the description of the TVT technique as a therapy to stress urinary incontinence, in 1996, about 150000 subjects have undergone it. This technique was first used in our centre in november 1998. This article is aimed to contain our view of its evolution in the past three years.

MATERIALS AND METHODS: 142 patients of an average of 59 years old have been operated on. In 57\% of the cases, we also focused on the solutions to anatomic pelvis disorders.

RESULTS: After a follow-up of 17 months and a mean of 14 months, $93 \%$ of the cases succeeded. Failures arose during the first six months of therapy. We found the following complications: $4.8 \%$ of bladder perforations, $17 \%$ of postoperative retentions of no longer than 30 days, $2 \%$ of long-term retentions, $2.7 \%$ of postsurgical hematomas, and $9 \%$ of urge incontinence. Surgery was performed on 10 patients who had undergone surgery against bladder incontinence before, and all the cases proved success.

CONCLUSIONS: This technique is simple and only requires a short surgical time, and it can be applied for major ambulatory surgery. Although it is possible to encounter complications, they rarely occur and results are still encouraging. However, it is now necessary to verify that our long-term studies match with the success rates of $84.7 \%$.

KEY WORDS: TVT. Stress urinary incontinence. 
$\mathrm{E}$ procedimiento denominado TVT para el tratamiento de la incontinencia urinaria de esfuerzo se basa en una nueva teoría sobre los mecanismos de cierre uretral en la mujer: la teoría integral, según la cual se cierra la uretra en la porción media. La falta de soporte en la uretra media por los ligamentos pubouretrales y el deterioro de la función e inserción de los músculos pubococcígeos predisponen a las pacientes a presentar IUE ${ }^{1,2}$.

Desde su descripción e introducción en la práctica clínica en 1995-1996 en los países nórdicos (Dr. Ulmsten), se han colocado unas 150.000 unidades siendo en Europa y fundamentalmente en Francia (40.000) y Alemania (20.000) donde más mujeres han sido tratadas ${ }^{19}$.

A finales de 1998 iniciamos en nuestro servicio la colocación de TVT para el tratamiento de la incontinencia de esfuerzo. Es un estudio retrospectivo en el que analizamos los resultados en estos tres primeros años en los que hemos intervenido a 142 pacientes.

\section{MATERIAL Y MÉTODOS}

Entre noviembre de 1998 hasta noviembre de 2001 hemos tratado a 142 pacientes que presentaban incontinencia de esfuerzo. El 87,5\% presentaban IUE pura y el resto incontinencia urinaria mixta. La edad mediana fue de 59 años (rango entre 32-81), la mediana en el número de partos vaginales es de 3 hijos (rango entre 9-0) y la mediana del seguimiento fue 14 meses. La duración de los sintomas antes de consultar al especialista fue una mediana de 5 años con un rango entre 1 año y 50 años.

Todas las pacientes fueron sometidas a historia clínica (Fig. 1) y exploración física en la que la exploración ginecológica incluía la existencia de cistocele, rectocele, colpocele, prueba de esfuerzo, test de Boney y función de los músculos pelvianos.

Dentro del estudio preoperatorio se incluía cistomanometría a aquellas que presentaban incontinencia mixta y/o antecedentes de cirugía previa anti-incontinencia. No se ha practicado este estudio en los casos de incontinencia de esfuerzo pura.

Consideramos como incontinencias de mínimos esfuerzos aquellas que se producen al andar;

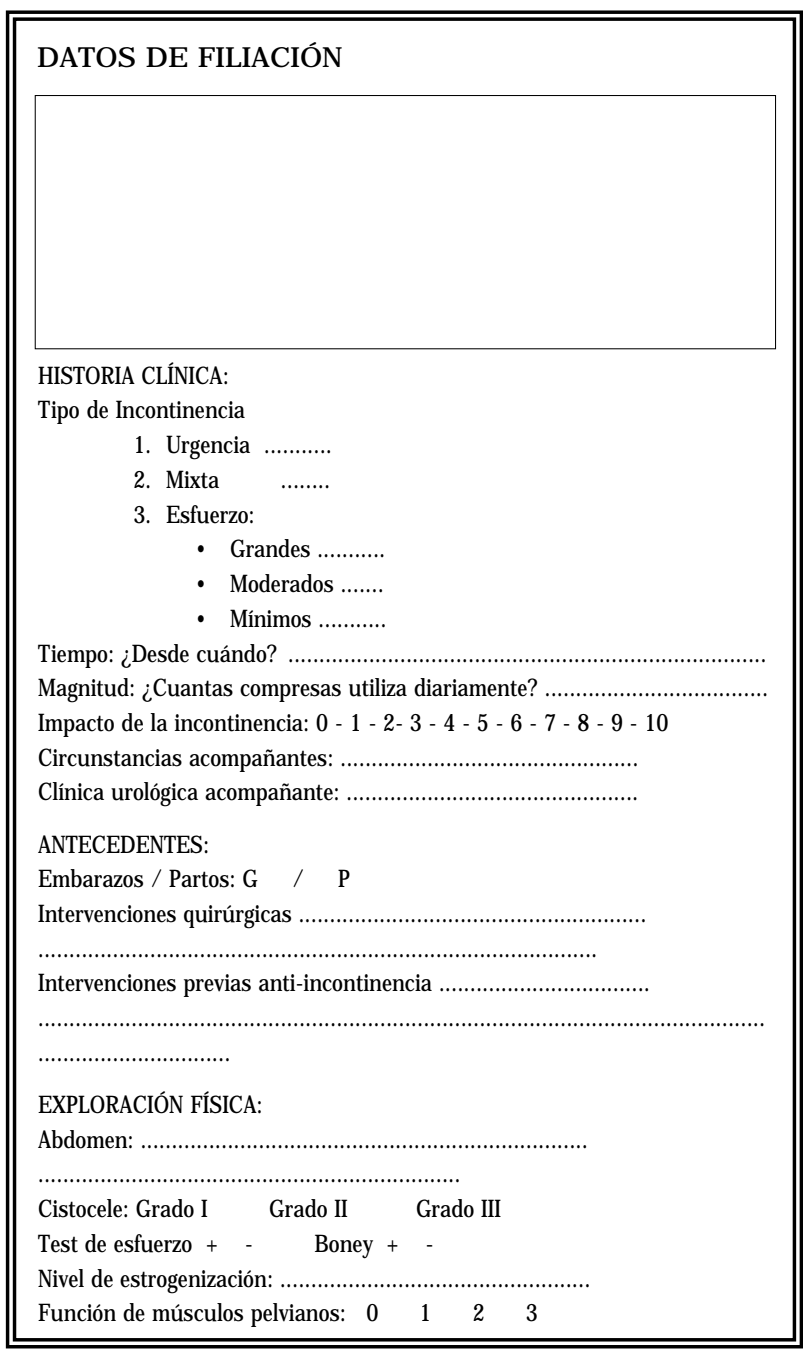

FIGURA 1

moderadas aquellas que tienen lugar cuando tosen o rien y de grandes esfuerzos cuando aparecen al coger pesos importantes o aparecen al hacer ejercicio físico (Tabla I), aunque reconocemos que esta clasificación es arbitraria y subjetiva, nos da una idea del grado de incontinencia y no utilizamos el "pad test" ya que creemos que es también subjetiva su valoración y mucho más engorrosa.

\section{TABLA I}

\begin{tabular}{|l|c|}
\hline Grandes esfuerzos & $6,6 \%$ \\
\hline Moderados esfuerzos & $33,5 \%$ \\
\hline Mínimos esfuerzos & $45,5 \%$ \\
\hline Incontinencia mixta & $12,5 \%$ \\
\hline
\end{tabular}


Dentro de la exploración física damos mucha importancia a la valoración del cistocele, ya que según su cuantificación tendrá implicaciones quirúrgicas. Consideramos cistocele grado I cuando no alcanza el introito, grado II cuando éste llega hasta introito vaginal y grado III cuando pasa más allá del introito vaginal ${ }^{3}$.

La valoración del colpocele la realizamos nosotros y si observamos que presenta colpocele grado II o mayor enviamos a la paciente al Servicio de Ginecología que son los que indican y realizan la histerectomía si procede.

\section{Procedimiento quirúrgico}

Con la paciente en posición ginecológica colocamos sonda vesical no 16 . Después realizamos dos incisiones de aproximadamente $0,5 \mathrm{~cm}$ justo por encima de la sínfisis de pubis y una en cara anterior de vagina suburetral a $0,5 \mathrm{~cm}$ de meato uretral externo y de una longitud de 1-1,5 cm. Posteriormente se procede a una disección de la fascia periuretral subyacente y de los espacios parauretrales hasta alcanzar la fascia endopélvica, sin perforarla y localizándola digitalmente.

A través de la sonda vesical introducimos una guía tutor rígida, con lo que podemos desplazar la uretra y el cuello vesical durante la inserción de la aguja.

Desplazando la uretra al lado contrario al que vamos a pasar la aguja (aguja y sonda se cruzan) pasamos ésta desde la apertura vaginal hasta hipogastrio, repitiendo la maniobra en el lado contralateral.

Con las agujas colocadas realizamos cistoscopia para comprobar integridad vesical ${ }^{4,15}$.

Durante este último año hemos decidido realizar el procedimiento con el cistoscopio colocado desde el principio y éste es el que desplaza la uretra y cuello vesical cuando introducimos las agujas, de tal forma que nos evitamos los pasos de la colocación de sonda, el paso de la guía tutor, retirar sonda y la introducción del cistoscopio (con esta forma de actuación nos ahorramos entre 5 y 10 minutos de cirugía).

Tras la extracción de ambas agujas por hipogastrio la cinta de poplipropileno queda alojada a nivel suburetral (en tercio medio). Se retira la envoltura de plástico que recubre la cinta (tras los primeros meses de realización de la técnica observamos que 4 pacientes presentaban retenciones urinarias que requerian autosondajes por lo que decidimos que en este paso quirúrgico quizás la cinta de polipropileno comprimía en exceso la uretra al retirar el plástico por lo que, desde entonces, cuando retiramos el plástico colocamos una tijera de Mayo entre la cinta y la uretra de tal forma que la cinta no la comprima) (Fig. 2).

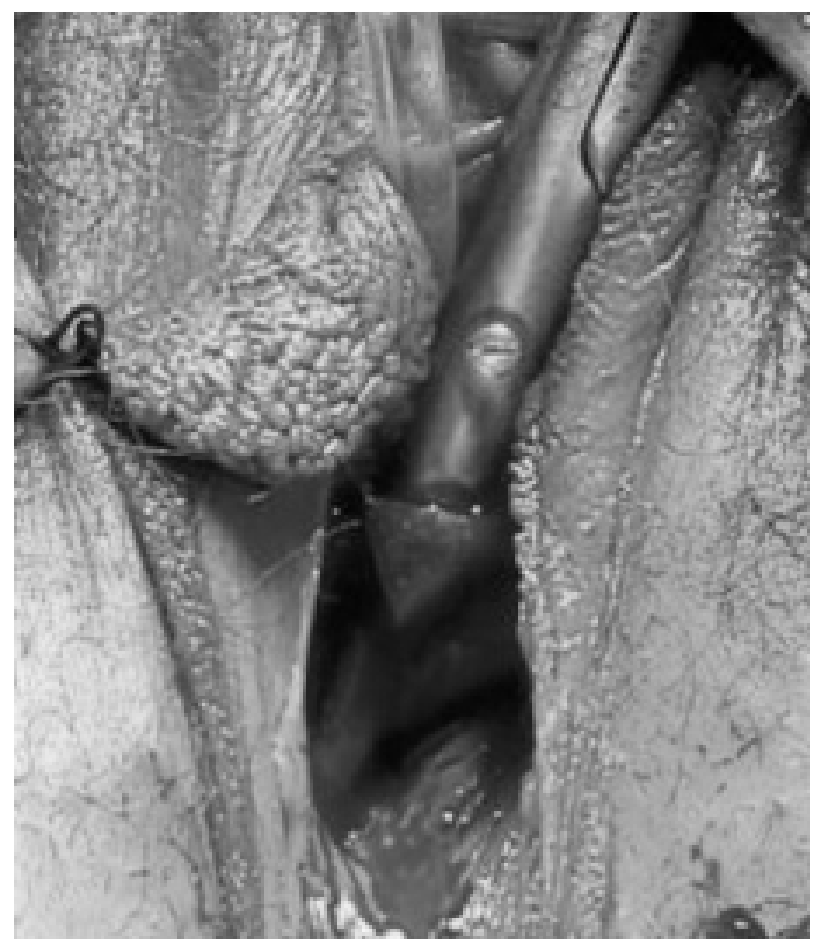

FIGURA 2

Aquellas pacientes que presentaban cistocele grado II o mayor en el mismo acto quirúrgico se realizó la corrección del cistocele y/o colpocele en los casos en que era necesario. En cuatro pacientes con antecedentes de cirugía retropúbica antiincontinencia y al inicio de la serie, se les indicó colocación de TVT asociando laparotomía para rechazar vejiga y controlar visualmente el paso de las agujas por el espacio retropúbico y esto se realizó así por la posible perforación vesical repetida. Posteriormente, hemos realizado la colocación de TVT sin laparotomía (6 pacientes), a pesar de sus antecedentes de cirugía previa sin presentar complicaciones ni intraoperatorias ni postoperatorias (Tablas II y III).

La técnica quirúrgica es realizada por todos los miembros del Servicio. 
TABLA II

\begin{tabular}{|l|l|}
\hline Cistocele grado O & $29 \%$ \\
\hline Cistocele grado I & $21 \%$ \\
\hline Cistocele grado II & $25 \%$ \\
\hline Cistocele grado III & $23 \%$ \\
\hline
\end{tabular}

\section{TABLA III}

\begin{tabular}{||l|c|}
\hline TVT simple & $41 \%$ \\
\hline Plastia anterior vaginal más TVT & $36 \%$ \\
\hline Histerectomia más plastias vaginales más TVT & $17 \%$ \\
\hline TVT más plastia anterior y posterior vaginal & $2 \%$ \\
\hline TVT por laparotomía & $2 \%$ \\
\hline
\end{tabular}

Todas las pacientes fueron intervenidas con anestesia general o raquídea; ninguna fue realizada con anestesia local tal y como describe el Dr. Ulmsten.

Cuando las pacientes presentaban colpocele que requería histerectomía, éstas eran ingresadas en el Servicio de Ginecología, la histerectomía fue realizada por los ginecólogos y nosotros acudíamos a su quirófano para colocar la TVT tras la cirugía ginecológica. Estas pacientes estaban a cargo del Servicio de Ginecología que indicaba el tiempo de ingreso y ordenaba el alta.

Cuando realizamos TVT simple o asociado a corrección de cistocele ha habido una variación a lo largo de estos tres años: al principio las pacientes eran ingresadas en el hospital y permanecían en él hasta conseguir micción, posteriormente seguían ingresando pero eran dadas de alta a las 24 horas de la intervención sin sonda o con sondajes intermitentes, en el caso de ser retencionistas. Este último año realizamos la intervención en régimen de cirugía mayor ambulatoria de tal forma que las pacientes salen del hospital con sonda vesical, que es retirada entre las 24 y 72 horas en la consulta (por ello existe una gran variabilidad en la cantidad de días de permanencia de sonda vesical en el post-operatorio inmediato).

La evaluación post-quirúrgica incluyó datos sobre la cirugía, complicaciones intra y post-quirúrgica y los resultados de la cirugía. Las pacientes fueron valoradas a los tres, seis meses, al año y una vez al año, mediante historia clínica (valoración personal de su incontinencia y si requiere o no compresas) y exploración física que incluye valoración del cistocele, rectocele, colpocele y test de esfuerzo con vejiga llena.

\section{RESULTADOS}

Criterios de curación

Estimamos como curación objetiva la ausencia de pérdida de orina en la prueba de esfuerzo con vejiga llena y curación subjetiva aquella que refiere la paciente como ausencia de pérdida de orina ${ }^{10}$. Por lo tanto, consideramos como curación aquellas pacientes a las que tanto objetiva como subjetivamente no presentan escapes y no requieren compresas, y como fracaso cualquier grado de incontinencia de esfuerzo aunque fuese menor que la previa a la cirugía.

Ha sido posible evaluar a todas las pacientes excepto a una que falleció durante el seguimiento por un tromboembolismo pulmonar no secundario a la cirugía (seguimiento 30 meses y continente).

Las pacientes fueron portadoras de sonda vesical una mediana de 1 día, media de 2 con un rango entre 1-9 días.

La duración mediana del seguimiento fue de 14 meses (rango 2-39 meses).

$\mathrm{El}$ fracaso de la técnica se produjo en el $7 \%$ de las pacientes, apareciendo en todos los casos de forma precoz en el control posterior a la cirugía o en los 6 primeros meses.

Para analizar nuestros resultados decidimos dividir la serie en cuatro grupos según el tiempo de seguimiento:

Grupo 1. Aquellas con un seguimiento menor de 12 meses (58 pacientes, 40\%) (mediana de seguimiento 5 meses).

Grupo 2. Seguimiento entre 12 y 24 meses ( 42 pacientes, 29\%) (mediana de seguimiento 18 meses).

Grupo 3. Seguimiento entre 24 y 36 meses (39 pacientes, 27\%) (mediana de seguimiento 29 meses).

Grupo 4. Seguimiento más de 36 meses (4 pacientes, 2,7\%) (mediana de seguimiento 37 meses).

En el primer grupo apareció incontinencia en dos pacientes (3\%), en el grupo 2 en otras dos 
$(4,8 \%)$, en el grupo 3 en 5 (12\%) y en el grupo 4 en $1(25 \%)$. Todas las pacientes presentaron fracaso tal y como hemos reflejado anteriormente en los primeros 6 meses.

Las complicaciones intraoperatorias ocurrieron en el 6\% (3 perforaciones vesicales, 4 hematomas retropúbicos en Retzius, uno de ellos quirúrgico, 2 aperturas de la sutura vaginal y una infección de la herida quirúrgica (todas estas complicaciones ocurrieron en el grupo 3). Estos datos tienen clara relación con la curva de aprendizaje ya que el grupo 3 de seguimiento coincide con la incorporación de todos los miembros del servicio a realizar la técnica.

El 17\% requirieron autosondajes intermitentes entre 3 y 30 días y sólo el $2 \%$ ( 3 pacientes) han necesitado autosondajes durante más de 6 meses. Estas pacientes pertenecen al grupo 4 ( 2 de ellas) y una al grupo 3 con mayor seguimiento (34 meses). Esto nos llevó a cambiar algún aspecto en la técnica quirúrgica. Cuando extraíamos la cubierta de plástico que cubre la cinta de polipropileno, quizás comprimíamos demasiado la uretra, por lo que desde entonces decidimos colocar una tijera entre banda de polipropileno y uretra, de tal forma que cuando extraemos la cubierta de plástico y cortamos la cinta a nivel suprapúbico, no hacemos que ésta presione a uretra.

También hemos encontrado dispareunia en 3 pacientes y dolor suprapúbico persistente en otras 3. Una paciente presentó retención urinaria a los dos años de seguimiento que ha requerido la sección quirúrgica de la malla. Otra paciente presentó una cicatrización defectuosa de la vagina que hubo que resuturar a los tres meses de la cirugía, curiosamente esta paciente no ha presentado infección de la malla y tras la $2^{\text {a }}$ cirugía es continente.

La urgencia miccional de novo apareció en 14 pacientes (9\%) que han respondido de manera satisfactoria a los anticolinérgicos orales.

De nuestra serie el $12,5 \%$ de las pacientes (17) presentaban incontinencia mixta aunque su componente fundamental era el esfuerzo. De éstas en 10 que supone un 58\%, desapareció la urgencia miccional y en 7 (42\%) persistió la clínica.

Hemos intervenido a 10 pacientes que tenían cirugía retropúbica previa anti-incontinencia (a 4 de ellas se les realizó la intervención asociando laparotomía) y las 10 están continentes con un seguimiento mediano de 21 meses (rango entre 5 y 32 meses).

También hemos recolocado una TVT a una paciente a la que se le había colocado una TVT previa en otro servicio y en la exploración la malla estaba colocada en cuello por lo que se le colocó otra en uretra media sin retirar la previa, estando hoy la paciente continente.

\section{DISCUSIÓN}

El procedimiento de la cinta vaginal sin tensión (TVT) para el tratamiento quirúrgico de la incontinencia de esfuerzo femenina, se basa en una nueva teoría sobre los mecanismos del cierre uretral en las mujeres: la teoría integral descrita por Petros y Ulmsten en 1990, según la cual la uretra se cierra en su porción media y no en el cuello de la vejiga ${ }^{1,2}$. La falta de soporte de la uretra media por los ligamentos pubouretrales, de la pared vaginal anterior suburetral y el deterioro de la función y de la inserción de los músculos pubocccigeos predisponen a que las pacientes padezcan incontinencia de esfuerzo.

En la literatura médica actual existen más de 100 procedimientos quirúrgicos para el tratamiento de la IUE. En los últimos 20 años se ha visto el ascenso y la caída de muchos de ellos. Tan pronto aparece una nueva técnica, son muchos los cirujanos que se incorporan a ella y publican sus resultados preliminares que posteriormente no son confirmados a medio y largo plazo.

Desde que Ulmsten y sus colaboradores describieron la técnica por primera vez en 1996, los resultados preliminares se han ido corroborando en publicaciones posteriores con un seguimiento más largo. Los buenos resultados, $84,6 \%$ de curaciones, se mantienen hasta con 60 meses de seguimiento ${ }^{4,5}$.

Otro aspecto que consideramos importante, es si los buenos resultados obtenidos por el creador de la nueva técnica quirúrgica son obtenidos por otros grupos de trabajo y a este respecto observamos que los datos son reproducibles en todas las series, incluida la nuestra.

También debemos tener en cuenta las complicaciones tanto intraoperatorias como post-operatorias y compararlas con la serie del Dr. Ulmsten, que consideramos como referencia (Tabla IV): 
TABLA IV

\begin{tabular}{|c|c|c|c|c|c|}
\hline Serie & $\begin{array}{l}\text { Perforación } \\
\text { vesical }\end{array}$ & $\begin{array}{c}\text { Retenciones } \\
\text { post-operatoria }\end{array}$ & $\begin{array}{l}\text { Autosondajes } \\
\text { a largo plazo }\end{array}$ & Hematoma & $\begin{array}{l}\text { Urgencia } \\
\text { de novo }\end{array}$ \\
\hline Ulmsten & $0,7 \%$ & $4,4 \%$ (<4 días) & $0,7 \%$ & $3,3 \%$ & \\
\hline $\begin{array}{l}\text { Tamussino (Serie } \\
\text { Austriaca) }\end{array}$ & $4 \%$ & - & - & & \\
\hline Meschia (Serie Italiana) & $6 \%$ & & $0,5 \%$ & $2 \%$ & \\
\hline Souli et al & $10 \%$ & $10 \%$ & $1,7 \%$ & & \\
\hline Lebret et al & $22 \%$ & $13 \%$ & $3 \%$ & & \\
\hline Jeffry et al & $11,6 \%$ & $10 \%$ & $2,5 \%$ & & $25,9 \%$ \\
\hline Jacquelin et al & & & $6 \%$ & & $4,6 \%$ \\
\hline Nuestra serie & $4,8 \%$ & $17 \%$ & $2 \%$ & $2,7 \%$ & $9 \%$ \\
\hline
\end{tabular}

1. Perforaciones vesicales: el porcentaje varía entre el 4 y el 11,8\% (nuestro grupo 4,8\%) sensiblemente mayor que el grupo de referencia $(0,7 \%)$. Todos los autores refieren que esto es debido al periodo de aprendizaje.

2. Retenciones post-operatorias (aquellas que ocurren en los 30 primeros días): ocurre lo mismo que en el apartado anterior. El autor las refiere y presenta en el 4,4\%, con sondajes durante menos de 4 días y en el resto de series incluida la nuestra los porcentajes varían entre 10-17\%.

3. Autosondajes a largo plazo (aquellos que persisten durante más de 30 d): teniendo como referencia el 0,7\% del autor, la serie que más se acerca es el estudio multicéntrico italiano que presenta esta complicación en el 0,5\%, el resto variamos entre el 1,7\% y el 6\%. En nuestra serie esta complicación se ha presentado en 3 pacientes (2\%) y se produjo en las primeras pacientes que sometimos a esta técnica. Posteriormente introdujimos la variante en la técnica quirúrgica que ya hemos explicado y no hemos vuelto a presentar este problema.

4. Hematomas: en todos los grupos ocurren en proporciones parecidas que varían entre un $2 \%$ y un 3,3\%. En nuestro grupo se presentó en 4 pacientes y sólo una de ellas requirió drenaje quirúrgico. Durante la laparotomía se observó sangrado a nivel de vena de gran calibre en la fascia endopélvica que se suturó y no retiramos la malla, la paciente está continente.
5. Urgencia de novo: son pocas las series que recogen esta complicación y llama la atención como en el estudio publicado por Jeffri y cols., ésta se presenta en un 25,9\%, mejorando sólo el $51,7 \%$ con anticolinérgicos ${ }^{8}$. En nuestro estudio esta complicación se presenta en el 9\% y todas ellas responden a anticolinérgicos.

6. Otras complicaciones: se han descrito otras complicaciones como son cicatrización vaginal defectuosa (Meschia y cols. en el 0,5\%, nuestra serie en el 0,6\% que se resolvió realizando nueva sutura vaginal). Dispareunia en el $2 \%$ que ha desaparecido en el seguimiento y dolor a nivel hipogastrio en el 2\%. Otra complicación que nos ha ocurrido en una sola paciente y que no viene reflejada en la literatura es una retención urinaria en paciente intervenida 2 años antes que previamente se encontraba sin residuos y que le intentamos retirar sonda en repetidas ocasiones sin conseguir micción, por lo que requirió la sección quirúrgica de la malla y en estos momentos se encuentra continente y sin residuos.

Del análisis de estos datos podemos inferir que, aún considerando como baja la tasa de complicaciones, nuestro objetivo como el del resto de grupos debe ser el logro de unas tasas similares a las del Dr. Ulmsten. Los grupos que comiencen con esta técnica obtendrán posiblemente un porcentaje de complicaciones similares a los del resto de grupos ${ }^{6-10,14,16,17}$. 
Aunque la técnica original se propuso para el tratamiento de la incontinencia genuina de esfuerzo con mínima alteración del suelo pelviano, todas las series la asocian a reparación de los defectos anatómicos concomitantes en un porcentaje que varía entre $10 \%$ y el $57 \%$ de nuestro grupo.

Otro aspecto a tener en cuenta son los resultados obtenidos en pacientes con incontinencia de urgencia asociada a la de esfuerzo, obteniendo mejorías entre un $50 \%$ en el estudio multicéntrico Italiano, nuestra serie $58 \%$ y un $85 \%$ en la serie del Dr. Rezapour y Ulmsten. Estos autores realizan urodinámica previa a la cirugía y aquellas que presentan inestabilidad vesical manifiesta y/o volúmenes vesicales pequeños $(<200$ cc como principal causa del trastorno) no las consideran aptas para la colocación de TVT. Por ello, al seleccionar mejor el grupo de pacientes candidatas a la colocación de TVT, obtienen mejores resultados ${ }^{11}$.

Tal y como he comentado anteriormente, aunque la técnica no estaba pensada para pacientes con antecedentes de cirugía anti-incontinencia pelviana, tanto Ulmsten como Rezapour y otros autores han obtenido excelentes resultados que varían entre $74 \%$ al $81 \%$. Nuestra experiencia es corta, de tan sólo 10 pacientes y todas ellas en el momento actual están continentes ${ }^{12,13,20}$ (Tabla V).

\section{TABLA V}

\begin{tabular}{||l|c|c|c||}
\hline Serie & Pacientes & $\begin{array}{c}\text { Seguimiento } \\
\text { medio }\end{array}$ & Éxito* \\
\hline Ulmsten et al. & 90 & 60 meses & $84,7 \%$ \\
\hline Ulmsten et al. & 50 & 24 meses & $92 \%$ \\
\hline $\begin{array}{l}\text { Tamussino } \\
\text { (Serie Austriaca) }\end{array}$ & 806 & 12 meses & $90 \%$ \\
\hline $\begin{array}{l}\text { Meschia } \\
\text { (Serie Italiana) }\end{array}$ & 429 & 21 meses & $92 \%$ \\
\hline Souli et al. & 120 & 15,2 meses & $86,7 \%$ \\
\hline Lebret et al. & 100 & 12 meses & $97 \%$ \\
\hline Liapis et al. & 68 & 24 meses & $90 \%$ \\
\hline Jeffry et al. & 112 & 25 meses & $89,3 \%$ \\
\hline Jacqueline et al. & 400 & 24 meses & $89,1 \%$ \\
\hline Nuestra serie & 142 & $\begin{array}{c}17 \text { meses } \\
\text { (mediana } 14)\end{array}$ & $93 \%$ \\
\hline
\end{tabular}

*Éxito: Curación objetiva y subjetiva de la incontinencia

\section{CONCLUSIONES}

Se trata de una técnica quirúrgica sencilla, que como toda técnica, tiene una curva de aprendizaje, que requiere un corto tiempo quirúrgico (aproximadamente 30 minutos) y que puede ser realizada en régimen de Cirugía Mayor Ambulatoria.

Es una técnica no exenta de complicaciones aunque la proporción de ellas es baja y con unos resultados que siguen siendo alentadores (aunque debemos esperar que nuestros estudios a largo plazo coincidan con los ya publicados con tasas de éxito del 84,7\%).

\section{REFERENCIAS}

1. PETROS P, ULMSTEN U.: An integral theory of female urinary incontinence. Experimental and clinical considerations. Acta Obstet Gynecol Scand 1990; 153: 7-31.

2. PETROS P, ULMSTEN U.: An integral theory and its method for the diagnosis and management of female urinary incontinence. Scand J Urol Nephrol 1993: 7-31

3. BADEN WF, WALKER TA.: Genesis of the vaginal profile: a corretale classification of the vaginal relaxation. Clin Obstet Gynecol 1972; 15: 1048-1054.

4. ULMSTEN U, FALCONER C, JOHNSON P.: A multicenter study of TVT for surgical treatment of stress urinary incontinence. Int Urogynecol $J$ 1988; 9: 210-213.

5. NILSSON I, KUUVA N, FALCONER C, REZAPOUR M, ULMSTEN U.: Long-term results of the tension free vaginal tape (TVT) procedure for treatment stress urinary incontinence. Internation Urogynecology Journal 2001; Supplement 12 (4); 65-68.

6. LEBRET T, LUGANE P.: Evaluation of tension free vaginal tape procedure. Its safety an efficacy in the treatment of female stress urinary incontinence during the learning phase. Eur Urol 2001 nov; 40 (5): 543-547.

7. LIAPIS A, BAKAS P.: Management of stress urinary incontinence in women with the use of TVT. Eur Urol 2001 nov; 40 (5): 548-551.

8. JEFFRY L, DEVAL B.: Objetive and subjetive cure rates after tension free vaginal tape for treatment urinary incontinence. Urology 2001; 58 (5): 702706.

9. GORDON D, GOLD R.: Combined genitourinary prolapse repair and prophilactic tension free vaginal tape in women with severe prolapse and occult stress urinary incontinence: preliminary results. Urology 2001 oct; 58: 547-550.

10. MESCHIA M, PIFAROTTI PT.: Tension free vaginal tape: analysis of outcomes and complications in 404 stress incontinent women. International Urogynecology Journal and Pelvic florr Dysfunction 2001; 12 (Suppl. 2): S2-S27. 
11. REZAPOUR M, ULMSTEN U.: Tension free vaginal tape (TVT) in women with mixed urinary incontinence. A long term folow up. International Urogynecology Journal and Pelvic florr Dysfunction 2001; 12 (Suppl. 2): S15-S18.

12. REZAPOUR M, ULMSTEN U.: Tension free vaginal tape (TVT) in stress women with intrinsic sphinter deficiency (ISD). A long term follow up. International Urogynecology Journal and Pelvic florr Dysfunction 2001; 12 (Suppl. 2): S12-S14.

13. REZAPOUR M, ULMSTEN U.: Tension free vaginal tape (TVT) in women with recurrent urinary incontinence. A long term follow up. International Urogynecology Journal and Pelvic florr Dysfunction 2001; 12 (Suppl. 2): S9-S11.

14. SOULIE M, DELBERT JULHES F.: Cure d`incontinence urinaire feminine par bandelette de prolene TVT: results preliminaries dune enquete multicentrique et prospective. Prog Urol 2000 sep; 10 (4): 622-628.

15. JIMÉNEZ CALVO, HUALDE A, SANTIAGO A.: TVT (cinta libre de tensión) nueva técnica quirúrgica para el tratamiento de la incontinencia urinaria de esfuerzo. Arch Esp Urol 2000 jan-feb; 53 (1): 9-13.
16. JACQUELIN B.: Utilisation du TVT dans la chirurgie de l'incontinence urinaire feminine. $J$ Gynecol Obstet Biol Reprod (París) 2000 may; 29 (3): 242247.

17. TAMUSSINO K, HANZAL E.: Registro austriaco de la cinta vaginal sin tensión. Urogynecology Journal and Pelvic florr Dysfunction 2001; 12 (Suppl. 2): S29.

19. Urología al día. TVT or not TVT. Rev Urol 2001; 2 (3): 126-128

20. AZAM U, FRAZER E.: The tension free vaginal tape procedure in women with previous failed stress incontinence surgery. $J$ Urol 2001 august; 166 (2): 554-556.

Dr. J. Jiménez Calvo

Plza. de los Castaños, 1 - 6으

31010 Barañaín (Navarra)

(Trabajo recibido el 4 marzo de 2003) 